\title{
Continuous Pain Related Behavior Recognition from Muscle Activity and Body Movements
}

\author{
Rui Qin ${ }^{1}$ and Hongying Meng ${ }^{1}$ \\ ${ }^{1}$ Dept. of Electronic and Computer Engineering \\ Brunel University London \\ Uxbridge, UB8 3PH, UK
}

\author{
Maozhen $\mathrm{Li}^{1,2}$ \\ ${ }^{2}$ The Key Laboratory of Embedded Systems and Service \\ Tongji University \\ Shanghai 200092, China
}

\begin{abstract}
Chronic pain is a disease that the patients suffers a lot in their daily life and it is difficult to be released completely. It is difficult to manage because pain can come anytime and it is unpredictable. However, the pain can be represented by the pain related behaviors such as guiding and abrupt actions. In this paper, we will develop a machine learning based system that can detect the pain related behaviors from patient's Electromyography (EMG) signals and body movements continuously. The system includes data collection, feature extraction, modeling and classification. The data were collected using biosensor sensor for EMG and motion capture for body movement. Specific features are extracted from the body movement data. Then Random Forest and a Two Stage Classification (TSC) scheme (KNN coupled with Hidden Markov Model (HMM)) were used for pain related behavior detection in a continuous manner. The proposed method was tested on EmoPain corpus dataset provided by UCL and experimental results demonstrate the efficiency of the proposed method.
\end{abstract}

\section{Keywords- EMG, Body movement, $k$-NN, HMM, Pain}

\section{INTRODUCTION}

Chronic Pain is neuropathic nociceptive last for a long time; usually last for more than 3 or 6 months with an arbitrary interval since onset [1]. Some studies of Epidemiological show that $10 \%$ to $55 \%$ people in the world have been plagued by chronic pain [2]. Chronic pain can originate from inner brain, neuro or inner body and very hard to treat. It is often been handled by professional pain management team including medical practitioners, clinical psychologists and many other professionals [3]. Some kind of chronic pain can be relieved by nonpaid medicines and psychological treatments, but they only effective for part of patients [4][5]. Chronic pain, especially from heart system or respiratory system, has been indicated increase the 10 year mortality. Chronic pain has been also proved to cause depression, anxiety, sleep disturbances, and neuroticism [6].

Chronic lower back pain (CLBP) is a lower back pain (LBP) disorder involving the muscles, nerves, and bones and usually last for more than 12 weeks [7][8]. CLBP is one of the most common kinds of chronic pain and is more common in elder people. LBP, pretty part of it is CLBP also causes large number of disabilities both in North American and Europe [9]. And there is still no effective method to prevent CLBP [10] although some studies show exercise appears to be useful [11].
To recognize and detect CLBP continuously is the first step of pain management. The automatic detection of CLBP can provide a long term, continues detect of chronic pain and supplement of medical treatment. This is a research area strongly related to emotion detection and affective computing. The behavior of reflections of chronic pain are including guarding, hesitation, bracing, abrupt action, limping and rubbing. Any of these can illustrate the existing of chronic pain [12].

There are several methods to detect CLBP, including facial express recognition [13], EMG signal collection, voice analysis [14] and motion capture. Though all these collected dataset, the unnatural way of body movement and facial expression can be recorded and analysis, like guarding behavior when touched sore spot or support the pain waist with arms when stand up.

The goal of this research is to automatically and continuously detect CLBP related behavior in daily life. For all the data has been collected, the facial expression and voice signal collected and analysis is very inconvenient and operability is poor for CLBP. CLBP detection by musculoskeletal pain is more practicability [15]. To achieve that, body movement and EMG should be collected at all time and continuously. There are already many wearable motion capture devices and wireless EMG sensors available in the market.

In order to recognize CLBP related behaviors in real-time and automatically though motion capture and EMG signals, machine learning can be used to build the model and make the predictions. This research leads an attempt of long-term continuous self-management for chronic pain patients [16].

The paper is organized as the following. In section 2, some related works are introduced. In section 3, the detailed information on the dataset, feature extraction, labels and machine learning methods are given. Section 4 is experimental results and section 5 concludes the paper.

\section{RELATED WORK}

Pain detection from machine learning methods has been studied extensively in recent years. Lucey et. al.[17] studies the automatic detection of shoulder pain using Facial Action Coding System (FACS). The pain information is associated frame by frame in a total recording of 200 videos. Then, Active Appearance Model (AAM) was used for the feature 
extraction and machine learning method was used for 2 classes (pain/no pain) classification.

Recently, Emo-Pain corpus database [18] has been collected in University College London which includes facial expression, body movement and EMG signals.

Aung et al [19] did some early work on the Emo-Pain corpus dataset using Random Forest (RF) to detect guarding behavior of the patients. Though the existence of the guarding behavior, chronic pain can be ascertain. Data used in [19] include joint angles, joint energy and EMG.

In [18], Aung studied different body movements like one leg stand, reach forward. The detection is based on the behaviors including guarding, support, hesitating, etc. Aung adds all labels of raters together and normalizes the labels into the range of $[0,1]$ for Random Forest $(\mathrm{RF})$ based regression. Among all the behaviors related to CLBP, Aung tested several behaviors with guarding, abrupt motion and limping.

Recently, Olugbadel [20] uses just part of the Emo-Pain corpus dataset with a focus on only one body motion, reach forward. Instead of using all the features of body motion, Olugbadel uses very limit part of body motion, like neck combine with left arm. This is not a real-time detection system, it is based on a whole instance and uses a twin slidewindow to analyze all frames of the instance and determine the chronic pain level of the instance.

In this paper, we will do further work to build the automatically detection of CLBP related behavior like guiding in some exercises such as one leg stand, moving forward, etc.

\section{METHODOLOGY}

In this work, we will select different subset from Emo-Pain Corpus Dataset and build the system for continuously detection of CLBP related behaviors.

The machine learning system is based on the continuously classification method on affective emotion level prediction proposed in [21]. In [21], a continuous multi-stage level classification method was proposed based on k-NN in the first stage and a HMM in the later stages. It has been used for facial expression and vocal expression prediction on the frame level with good results. Here, we use k-NN in the first stage, and then extend the HMM in the later stage and make it a Two Stage Classification (TSC) scheme. The results were compared with the one from RF on the frame level.

\section{A. Emo-Pain Corpus Dataset}

The data collection includes two parts: the facial express is collected by 8 cameras around the experimental site, this part of videos watched by several psychologist and give a mark as possibility of CLBP and then used as labels; another part is motion capture and EMG collected by wearable device on subject participant who is asked to move and do different movement in a 5 meters long walking and motion capture area and the captured motion and EMG is used as features.

Body movement was captured by a customized motion capture Animzaoo IGS-190 suit which has eighteen small solid-state inertial sensors to accurately measure the exact rotations in real-time. The suit is comfortable to wear and minimal obstruction of movement when measuring data. EMG is collected by BTS FREEEMG 300. It has four wireless sensors, the range of probes and receivers can up to 50meters and also contains a BTS EMG-Analyzer. In this case, two probes are placed on the trapezius muscles and another two are placed on paraspinal muscles.

The behaviors of chronic pain in Emo-Pain corpus include guarding, hesitation, support, abrupt action, limping and rubbing. Here we focus on guarding and abrupt action behavior. Guarding is a stiff, interrupted or rigid movement, like prevent pain point of body contact with chair when sit down. Abrupt action is a sudden movement extraneous to the intended motion, which is a lack of body control cause by chronic pain.

The labels are produced by 4 psychologists though watch the facial expression frame by frame. Each of them watches the video and uses a specially designed remote controller to give a continuously score in the range of $[0,1]$. Each score represented a possibility of one behavior of CLBP. In the EmoPain corpus dataset, the continuously scores has already been translate to a discretization labels, $0,1,2$ means no behaviors of CLBP, possible have behaviors of CLBP, have behaviors of CLBP respectively.

There are four scores from four raters for each frame; we use a majority voting tactics to transfer four scores into one label. The final three classification labels are also $0,1,2$ respectively representation as no behaviors of CLBP, possible have behaviors of CLBP, have behaviors of CLBP. Specifically, when added the labels of 4 raters together, the sum is 0 or 1 , the final label is 0 , the sum is 2,3 , and 4 , the final label is 1 , the sum is 5 or larger, and the final label is 2 . The classification label divided is based on voting principle, when half of the raters think it is possible, the final label is considered as possible, and when all raters think it is possible and one of them think it is confirmed, or more than half of raters think it is confirmed, the final label is determined as having this behavior of CLBP. So the problem becomes a 3class classification problem.

\section{B. Features}

The dataset of body movement and EMG is collected from 22 participants with widely ages and both genders. Each participant have done different exercise for several times including one leg stand, sitting still, reach forward, sit to stand, stand to sit, bend and walk. 


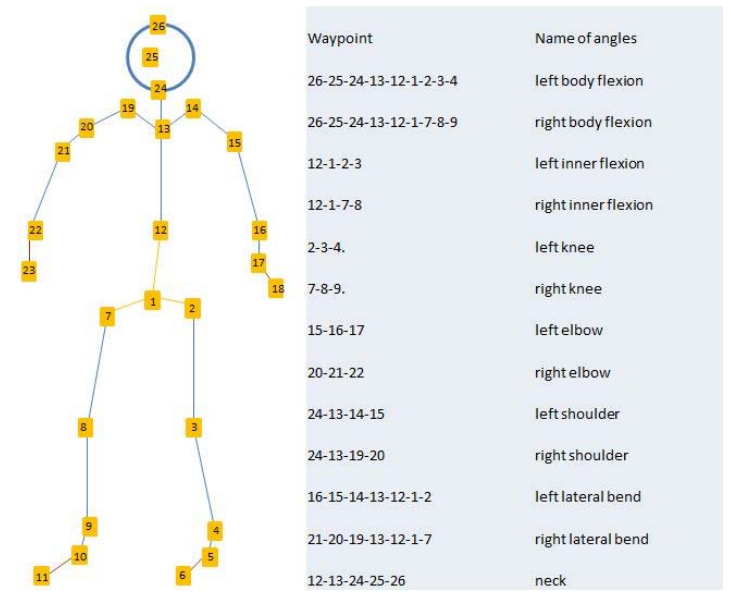

Figure 1. The joint angles used as features

There are 26 points and $78 \mathrm{XYZ}$ parameters totally in the coordinate's data. According to the motion capture data, there are 13 angles totally. The names of angles and the position of each point of angles are shown as Figure 1. These values are the feature vector in machine learning process. At the same time, 4 channels of EMG data were collected.

With the 78 space coordinates and 13 angles, different features can be calculated. All the features in the Emo-Pain corpus dataset are shown as Table I below, including angles values of 13 Angles, energy per joint, Euclidean distances travelled for 25 global trajectories and 24 relative trajectories to the hip node, spectral Arc Length for 25 global trajectories and 24 relative trajectories over each exercise type, directness Index of 25 global trajectories and 24 relative trajectories over each exercise type, and movement size for 25 global and 24 relative trajectories over each exercise type.

TABLE I. FEATURES OF EMO-PAIN CORPUS

\begin{tabular}{|l|c|}
\hline \multicolumn{1}{|c|}{ Features } & Number of features \\
\hline Joint Angle & 13 \\
\hline Joint Energy & 13 \\
\hline Speed(Distance between samples) & 49 \\
\hline Trajectory Smoothness(Spectral Arc Length) & 49 \\
\hline Trajectory Directness & 49 \\
\hline Movement Size(Alpha Volume) & 49 \\
\hline EMG(Electromyography) & 4 \\
\hline
\end{tabular}

\section{Classification}

For the classification, we divide the 22 participants into 3 groups for 3 -folds cross-validation. Each time we use 2 groups for training and 1 group for testing.

The whole system is shown in Figure 2. Before classification, the original data is divided into training part and testing part as mentioned before. We do the classification on the frame level using both TSC and RF for the comparison.

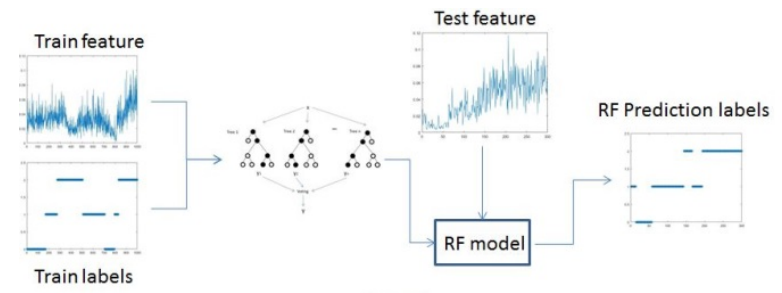

(a) $\mathrm{RF}$

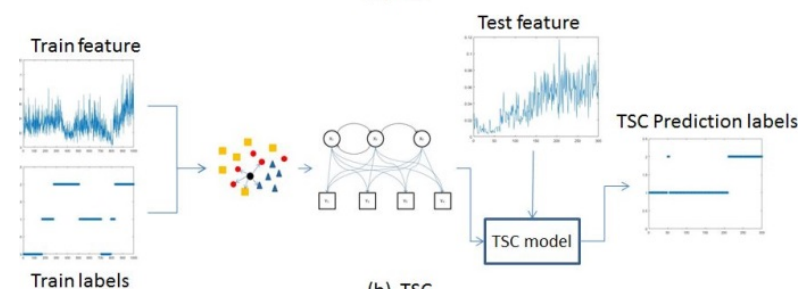

(b) TSC

Figure 2. Flow diagram for classification. (a). RF (b). TSC

1) $R F$

In $R F$, a subset of features in the training dataset is used to determine a node of decision tree; then sampling from the dataset for several times to constitute a training set, and using leftover samples as test set to assess the errors; for every node of the decision tree, randomly chosen some features and all the nodes are determined by these features; according to these features, calculate the best way of produce the decision tree and build a complete tree classification.

The advantages of RF are including the highly accuracy of classification it provides and large number of input variables it can process. It can also assess the importance of parameters in determining categories. It can be generalized within the error after not produce an estimate of bias when the construction of the forest. It also contains a good way to estimate the missing data and can still maintain accuracy when lost part of the information. RF provides an experimental method to detect variable interactions and can balance error for unbalanced classification data set.

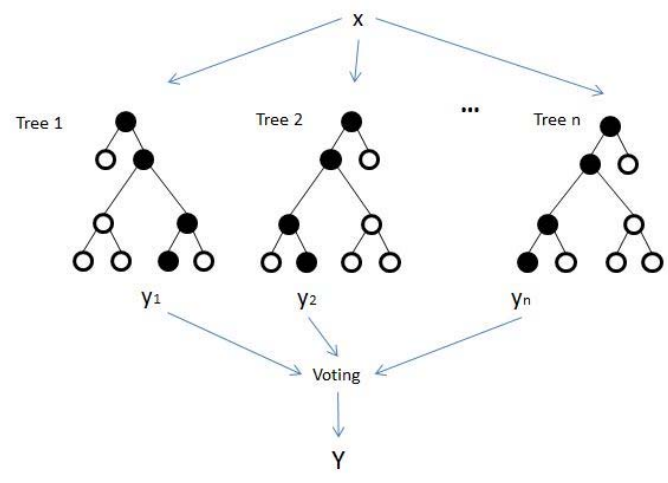

Figure 3. Diagram of RF

2) Two-stage classification (TSC)

As mentioned before, this TSC method contains two part of classification, k-NN and HMM. Firstly, k-NN is used for a prediction, and then HMM is used to learn the relationship between these predictions and re-fine the prediction labels of $\mathrm{k}-\mathrm{NN}$ based on possibility of states change. 


\section{a) $K N N$}

$\mathrm{K}-\mathrm{NN}$ algorithm is a very stable and reliable classification method in machine learning area. When training a feature with $\mathrm{k}-\mathrm{NN}$, it firstly produce an $\mathrm{N}$-dimension space, $\mathrm{N}$ is decided by the number of features. In this $\mathrm{N}$-dimension space, every sample has a label. When it predicts test dataset, this algorithm will consider several points have been training which are nearest to this test data point. The number of the points which have been chosen is $\mathrm{K}$ and among all the trained points, which label has appear most times will be predicted as the class of the test data. $\mathrm{K}$ usually is an odd number when in a classification situation to avoid two classes have the same votes. And to make the boards of each class more clearly, the smaller $\mathrm{K}$ has advantage.

$\mathrm{K}-\mathrm{NN}$ is a stable and reliable algorithm for classification, but comparing with RF which is also one of the most common used classification methods, the performance of original k-NN usually not as well as that of RF. But considering the calculate speed, k-NN is much faster than that of RF. In summary, original k-NN is stable and fast, but as lack of verification and bootstrap sample process, the performance of it usually is not as good as RF. So we decide to modify the last step of k-NN, when determine the class the test data, the original k-NN use a vote method, choose the most labels of train data.

\section{b) $H M M$}

HMM is a static model to describe Markov process contains implied unknown parameter. The hidden states of HMM are not directly visible, but some variables influenced by states are visible. It will be used in the decision level in the following.

\section{c) $\mathrm{TSC}$}

There are two stages in our TSC system. At the first stage, $\mathrm{k}-\mathrm{NN}$ algorithm is used for all the features in the frame level and the predict labels will be generated as the decision values. These decision values can be treated as the time series where the relationship between consecutive frames can be modeled as a Markov process because the behavior happens slowly.

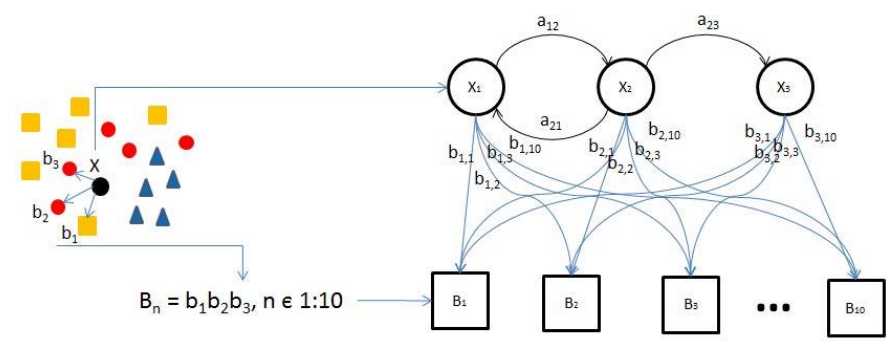

(a)

(b)

Figure 4. Diagram of TSC. (a). K-NN (b). HMM

In details, firstly, we use $\mathrm{k}=3 \mathrm{k}-\mathrm{NN}$ classifier to predict the labels and get the labels of 3 training samples nearest to the testing sample. As we mentioned before, there are three states 0,1 and 2 in labels. In HMM, these three states are $X_{1}, X_{2}$ and $\mathrm{X}_{3}$ in transition matrix. And for the 3 nearest labels of train set, there are 10 situations in total, so the HMM emission matrix is a 3 times 10 matrix, meaning under each $X_{n}(n=1,2$ or 3$)$, the possibility of each situation of 3 nearest samples' label. The whole TSC system is shown in Figure 4.

Matrixes below are examples of transition matrix and emission matrix. Equation 1 is transition matrix, each elements in it means possibility from previous state change to next state. In the table, $a_{m n}$ means the previous state is $m$, next state is $n$ and the possibility of $m$ change to $n$ is $a_{m n}$. Equation 2 is the emission matrix, each elements in it means possibility of combination of 3 nearest samples' labels. For $k=3$ situation, the 10 situations are $000,001,002,011,012,022,111,112$, 122 and 222. These situations are combined without orders. In the table, $b_{m, n}$ means the current state is $m$, the hidden situation is $\mathrm{n}$ and $\mathrm{b}_{\mathrm{m}, \mathrm{n}}$ is the possibility of $n$ in state $m$.

$$
\begin{aligned}
& \text { HMM Tansition Matrix }=\left[\begin{array}{lll}
a_{11} & a_{12} & a_{13} \\
a_{21} & a_{22} & a_{23} \\
a_{31} & a_{32} & a_{33}
\end{array}\right] \\
& \text { HMM Emission Matrix }=\left[\begin{array}{llll}
b_{1,1} & b_{1,2} & \ldots & b_{1,10} \\
b_{2,1} & b_{2,2} & \ldots & b_{2,10} \\
b_{3,1} & b_{3,2} & \ldots & b_{3,10}
\end{array}\right]
\end{aligned}
$$

\section{EXPERIMENTAL RISULTS}

In our experiments, k-NN, TSC, and RF methods are compared. Although there are lots of data in Emo-Pain corpus dataset and the behavior of labels are conclude guarding, support, abrupt action and hesitation. But for most of these behaviors, there are too many 0 labels and the classification of them seems pointless. Here we choose the part of data with most kinds of labels.

As mentioned before, we use a 3-folds cross-validation method on the testing. In the Emo-Pain corpus dataset, there are 22 participants, we divided them into three groups and each time, using 2 groups as training and 1 group as testing.

To calculate the accuracy of classification, we denote the values as $\mathrm{Y}_{\mathrm{mn}}$, for numbers of prediction in row $m$ and column $n$ in a confusion matrix. The accuracy can be calculated as by Equation 3 below.

$$
\text { Accuracy }=\frac{\sum_{n=1}^{3} Y_{n n}}{\sum_{m=1}^{3} \sum_{n=1}^{m} Y_{m n}}
$$

The classification results are shown in Table II below. The classification was done in 7 different situations with the combination of behavior and exercise. The results on k-NN, RF and TSC are compared.

\begin{tabular}{|c|c|c|c|c|c|}
\hline \multirow[t]{2}{*}{ Label } & \multirow[t]{2}{*}{ Exercise } & \multicolumn{3}{|c|}{ Classification Method } & \multirow{2}{*}{$\begin{array}{c}\text { Length of } \\
\text { samples }\end{array}$} \\
\hline & & K-NN & TSC & RF & \\
\hline Guarding & $\begin{array}{ll}\text { One } & \text { leg } \\
\text { stand } & \end{array}$ & $48.80 \%$ & $53.93 \%$ & $49.21 \%$ & 14225 \\
\hline $\begin{array}{l}\text { Abrupt } \\
\text { action }\end{array}$ & $\begin{array}{l}\text { One leg } \\
\text { stand }\end{array}$ & $37.58 \%$ & $56.50 \%$ & $53.27 \%$ & 14225 \\
\hline Guarding & $\begin{array}{l}\text { Reach } \\
\text { forward }\end{array}$ & $37.25 \%$ & $49.79 \%$ & $42.20 \%$ & 25214 \\
\hline Guarding & to & $42.92 \%$ & $31.52 \%$ & $52.28 \%$ & 4948 \\
\hline
\end{tabular}

TABLE II. ACCURACY AND LENGTH OF FEATURES UNDER THREE-FOLDS CROSS VILIDATION CLASSFICATION WITH TSC AND RF 


\begin{tabular}{|l|l|l|l|l|l|}
\hline & stand & & & & \\
\hline Guarding & $\begin{array}{l}\text { Stand to } \\
\text { sit }\end{array}$ & $45.56 \%$ & $44.70 \%$ & $\mathbf{5 8 . 4 9 \%}$ & 5236 \\
\hline Guarding & $\begin{array}{l}\text { Sit to } \\
\text { stand not } \\
\text { instruct }\end{array}$ & $45.14 \%$ & $33.86 \%$ & $\mathbf{4 5 . 9 4 \%}$ & 6004 \\
\hline Guarding & $\begin{array}{l}\text { Bend to } \\
\text { pick up }\end{array}$ & $40.31 \%$ & $\mathbf{4 2 . 4 3 \%}$ & $36.41 \%$ & 6037 \\
\hline
\end{tabular}

From Table 2, it can be seen that the performance of the classification is related to the length of the features (number of the samples in a time series). For short sequence, RF achieved the best results while for long sequences, TSC achieved the best performance. It can be explained that the HMM model need to be trained using long sequence because the model can be fully learned only with efficient samples. For the short sequence, RF is a better classifier than k-NN.

HMM gives each state a probability to change to the next state. So the continuously of the prediction series is better than $\mathrm{k}-\mathrm{NN}$ prediction series. And that is the reason of the better performance HMM has than $\mathrm{k}-\mathrm{NN}$ and $\mathrm{RF}$.

As HMM is a series based method, it works well only when the series are long enough. When the series is short, the result of HMM is very unstable and the result of RF is more stable when samples are limited.

It is obvious when the samples are long enough, more than ten thousands; the correct rate of TSC is higher than that of RF. But when the length of samples decreases to few thousands, the performance of TSC decrease significantly. In the four groups of data length less than 10 thousands, only one group's result shows TSC is better than that of RF.

In order to give more detailed information on the classification, Table 3 gives the confusion matrix obtained in the classification for Guarding action recognize in exercise Reach Forward under classification method of TSC. From this table, it can be seen that the majority of the labels are correctly classified.

TABLE III. CONFUSION MATRIX FOR GUARDING IN REACH FORWARD WITH TSC CLASSIFIER

\begin{tabular}{|l|l|c|c|c|}
\hline \multicolumn{2}{|c|}{} & \multicolumn{3}{|c|}{ Predict labels (Ymn) } \\
\cline { 2 - 5 } \multicolumn{2}{|c|}{} & No Guarding & Possible & Guarding \\
\hline \multirow{2}{*}{$\begin{array}{l}\text { Ture } \\
\text { labels }\end{array}$} & No Guarding & 2778 & 1541 & 2294 \\
\cline { 2 - 5 } & Possible & 2537 & 5109 & 3045 \\
\cline { 2 - 5 } & Guarding & 1628 & 1774 & 4508 \\
\hline
\end{tabular}

\section{CONCLUSIONS}

In this paper, an automatic CLBP related behavior detection system has been built from EEG and motion capture data from Emo-pain corpus database. The system can detect the behavior in a continuous way frame by frame.

Different machine learning methods have been used in our system. Although RF has been proved as a very effective method for classification, the performance of combination of TSC is still better in long sequences.

\section{ACKNOWLEDGMENT}

We would like to thank Dr. Min Aung and Professor Nadia Bianchi-Berthouze and others for the dataset.

This research is partially supported by the 973 project on Network Big Data Analytics funded by the Ministry of Science and Technology, China. No. 2014CB340404.

\section{REFERENCES}

[1] Turk, D.C.; Okifuji, A. (2001). "Pain terms and taxonomies". In Loeser, D.; Butler, S. H.; Chapman, J.J.; Turk, D. C. Bonica's Management of Pain (3rd ed.). Lippincott Williams \& Wilkins. pp. 18-25.

[2] Harstall C, Ospina M. How Prevalent Is Chronic Pain? June 2003 volume XI issue2 Pain Clinical Updates, International Association for the Study of Pain.

[3] Main, Chris J.; Spanswick, Chris C. (2000). Pain management: an interdisciplinary approach. Churchill Livingstone.

[4] "The effectiveness and risks of long-term opioid therapy for chronic pain: a systematic review for a National Institutes of Health Pathways to Prevention Workshop". Ann. Intern. Med. 162: 276-86. 2015.

[5] Tauben D (2015). "Nonopioid medications for pain". Phys Med Rehabil Clin N Am 26 (2): 219-48.

[6] Welsch P, Sommer C, Schiltenwolf M, Häuser W (2015). "[Opioids in chronic noncancer pain-are opioids superior to nonopioid analgesics? A systematic review and meta-analysis of efficacy, tolerability and safety in randomized head-to-head comparisons of opioids versus nonopioid analgesics of at least four week's duration]". Schmerz (in German) 29 (1): 85-95.

[7] "Low Back Pain Fact Sheet". National Institute of Neurological Disorders and Stroke. November 3, 2015. Retrieved 5 March 2016.

[8] Koes, BW; van Tulder, M; Lin, CW; Macedo, LG; McAuley, J; Maher, $\mathrm{C}$ (December 2010). "An updated overview of clinical guidelines for the management of non-specific low back pain in primary care.". European Spine Journal 19 (12): 2075-94.

[9] Manchikanti L, Singh V, Datta S, Cohen SP, Hirsch JA, ASIPP (2009). "Comprehensive review of epidemiology, scope, and impact of spinal pain". Pain Physician 12 (4): E35-70.

[10] Hoy D, Bain C, Williams G. A systematic review of the global prevalence of low back pain. Arthritis Rheum. June 2012, 64 (6): 202837.

[11] Steffens, Daniel; Maher, Chris G.; Pereira, Leani S. M.; Stevens, Matthew L; Oliveira, Vinicius C.; Chapple, Meredith; Teixeira-Salmela, Luci F.; Hancock, Mark J. (11 January 2016). "Prevention of Low Back Pain". JAMA Internal Medicine 176: 199-208.

[12] F.J. Keefe and A.R. Block. "Development of an observation method for assessing pain behaviour in chronic low back pain patients". Behaviour Therapy, 13(4), 1982.

[13] Kaltwang,S., Rudovic,O., Pantic,M., 2012. Continuous Pain Intensity Estimation from Facial Expressions. Advances in Vis. Comp. Springer, (2012), 368-377

[14] Romera-Paredes, B., Aung, M.S.H., Pontil, M.,Watson, P., Williams, A $\mathrm{CdeC}$, and Bianchi Berthouze, N., Transfer Learning to Account for Idiosyncrasy in Face and Body Expressions. IEEE FG'13, (2013).

[15] J.W.S. Vlaeyen and S.J. Linton. "Fear-avoidance and its conse-quences in chronic musculoskeletal pain: a state of the art". Pain, 85(3), 2000

[16] D.C. Turk and A. Okifuji, Psychological factors in chronic pain: evolution and revolution. J Consult Clin Psychol., 70(3), pp. 678-690, 2002.

[17] Lucey, J.F. Cohn, K.M. Prkachin, P.E. Solomon, and I. Matthews, "Painful data: The UNBC-McMaster shoulder pain expression archive database," in Proc. IEEE Int. Conf. Autom. Face Gesture Recog., Workshop , 2011, pp. 57-64. 
[18] Min S. H. Aung, Sebastian Kaltwang, Bernardino Romera-Paredes, Brais Martinez, Aneesha Singh, Matteo Cella, Michel Valstar, Hongying Meng, Andrew Kemp, Moshen Shafizadeh, Aaron C. Elkins, Natalie Kanakam, Amschel de Rothschild, Nick Tyler, Paul J. Watson, Amanda C. de C. Williams, Maja Pantic, and Nadia Bianchi-Berthouze, "The automatic detection of chronic pain-related expression: requirements, challenges and a multimodal dataset," IEEE Transactions on Affective Computing, 2015.

[19] Min S. H. Aung, Amanda C de C Williams, Aneesha Singh, Paul Watson, Soo Ling Lim and Nadia Bianchi-Berthouze, "Automatic
Recognition of Protective Behaviour in Chronic Pain Rehabilitation," 2013, Trento, Itlay.

[20] Temitayo A. Olugbade1, M. S. Hane Aung, Nadia Bianchi-Berthouze, Nicolai Marquardt, Amanda C. Williams "Bi-Modal Detection of Painful Reaching for Chronic Pain Rehabilitation Systems,'”CMI 2014.

[21] Hongying Meng and Nadia Bianchi-Berthouze "Affective state level recognition in naturalistic facial and vocal expressions," IEEE Transactions on Cybernetics, 44(3): 315-328. 